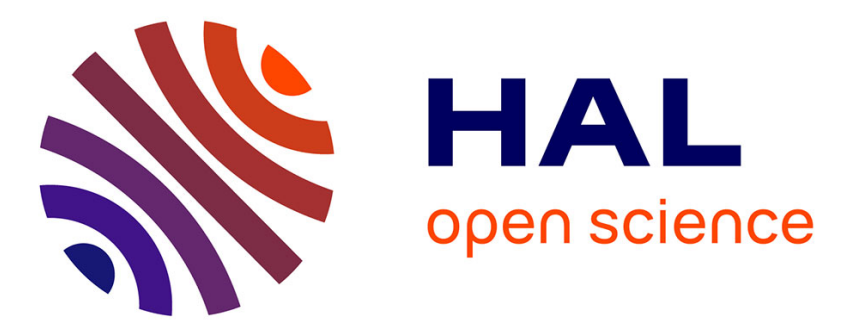

\title{
Avoir ou être ses pratiques sexuelles. Discours, performance et sexualité dans la théorie du genre de Judith Butler
}

\author{
Nathanaël Wadbled
}

\section{- To cite this version:}

Nathanaël Wadbled. Avoir ou être ses pratiques sexuelles. Discours, performance et sexualité dans la théorie du genre de Judith Butler. Rue Descartes, 2019, Discours, performance et sexualités Corpus, 95, pp.44-57. 10.3917/rdes.095.0044 . hal-02148227

\section{HAL Id: hal-02148227 \\ https://hal.science/hal-02148227}

Submitted on 16 Jun 2019

HAL is a multi-disciplinary open access archive for the deposit and dissemination of scientific research documents, whether they are published or not. The documents may come from teaching and research institutions in France or abroad, or from public or private research centers.
L'archive ouverte pluridisciplinaire HAL, est destinée au dépôt et à la diffusion de documents scientifiques de niveau recherche, publiés ou non, émanant des établissements d'enseignement et de recherche français ou étrangers, des laboratoires publics ou privés. 


\section{AVOIR OU ÊTRE SES PRATIQUES SEXUELLES. DISCOURS, PERFORMANCE ET SEXUALITÉ DANS LA THÉORIE DU GENRE DE JUDITH BUTLER}

Nathanaël Wadbled

Collège international de Philosophie | « Rue Descartes »

2019/1 N 95 | pages 44 à 57

ISSN 1144-0821

Article disponible en ligne à l'adresse :

https://www.cairn.info/revue-rue-descartes-2019-1-page-44.htm

Distribution électronique Cairn.info pour Collège international de Philosophie.

(C) Collège international de Philosophie. Tous droits réservés pour tous pays.

La reproduction ou représentation de cet article, notamment par photocopie, n'est autorisée que dans les limites des conditions générales d'utilisation du site ou, le cas échéant, des conditions générales de la licence souscrite par votre établissement. Toute autre reproduction ou représentation, en tout ou partie, sous quelque forme et de quelque manière que ce soit, est interdite sauf accord préalable et écrit de l'éditeur, en dehors des cas prévus par la législation en vigueur en France. Il est précisé que son stockage dans une base de données est également interdit. 


\section{NATHANAËL WADBLED}

Avoir ou être ses pratiques sexuelles. Discours, performance et sexualité dans la théorie du genre de Fudith Butler

\section{Être marqué par des pratiques discursives}

Lorsqu' elle définit l'identité de genre comme performative, Judith Butler lui donne la forme d'un discours par lequel elle se trouve identifiée et affirmée ${ }^{1}$. Il s'agit d'un ensemble d'éléments signifiants s'agençant selon une certaine structure dans un ensemble ou une composition qui tire elle-même sa signification autant de ces éléments que de leur agencement. L'identité de genre est alors la résultante d'éléments distinctifs se structurant selon une certaine grammaire et un certain usage. Ils ont la forme d'un ensemble de lois, qui est à la fois un répertoire, et qui différencient chaque individu de ceux adoptant un autre genre. Ces éléments n'ont pas seulement une forme langagière : ce sont des mots, mais aussi bien des gestes, des postures ou des attitudes qui prennent sens comme désignant ou, plus exactement, comme faisant signe vers une identité de genre. De la même manière que le nom propre reçu ou que l'accord des adjectifs et des participes, ces types de conduite constituent des marques indiquant et signifiant l'appartenance à un genre. Le genre est alors une stylisation langagière et corporelle qui identifie le sujet en tant que les spécificités d'un genre donné sont «marquées par des pratiques discursives ${ }^{2}$ ». Une telle conception place l'identité de genre du côté de la représentation ou de la performance. Il s'agit de prendre des postures linguistiques et physiques correspondant à une certaine identité. Pour comprendre ce qui se joue alors, Judith Butler attribue à l'énonciation genrée une fonction qui n'est ni constative ni dissimulatrice comme c'est le cas dans la performance théâtrale qui réalise un rôle se 
substituant à l'identité de l'acteur le temps de la représentation. Les énoncés genrés sont définis comme performatifs au sens où le sont des actes de langage, ce qui signifie que l'énonciation a pour fonction de produire un effet et que la performance a une dynamique rituelle. C'est dans cette perspective que Judith Butler définit la nature des énoncés genrés qu'assument les individus, et en particulier celle de leurs pratiques sexuelles.

\section{L'énonciation performative du genre}

\section{L'histoire du genre mise en mots et en gestes}

Définir l'identité de genre comme performative signifie que l'énoncé genré ne se contente pas de signifier une chose, mais réalise ce qu'il nomme, de sorte qu'il y a une apparente coïncidence entre signifier et agir ${ }^{3}$. L'identité est indissociable des énoncés qui la marquent. Le sujet genré n'a pas de statut ontologique indépendamment des actes qui constituent sa réalité. Il ne préexiste pas comme tel à son action et est donc un effet de son propre discours. Il ne précède pas ontologiquement ses différents rôles et fonctions par lesquels son identité prend sens et est socialement visible. Pour réaliser cet effet, l'énoncé doit être produit selon une forme déterminée. Les éléments énoncés ainsi que la manière dont ils le sont doivent respecter une norme contraignante et réglée. Ces mots et ces gestes s'inscrivent dans le cadre d'une définition normative de ce que doivent être les identités de genre et de la bonne manière de l'exprimer.

Cette situation se distingue de ce que serait une performance au sens d'un résultat ou d'une exécution théâtrale. D'un côté, l'expression du genre réalise une identité préalablement définie comme lorsqu'il s'agit de réussir à atteindre un objectif. Judith Butler se distingue de toute conception essentialiste de l'identité. Le discours genré que porte le sujet sur son corps et dans son langage n'est pas l'explicitation d'une nature psychique ou corporelle ${ }^{4}$. Sans affirmer qu'il n'y aurait ni psyché ni corps biologique propre avant les actes marquant son genre, Judith Butler considère que ces derniers non seulement définissent l'identité, mais plus encore la rendent effective. Le genre ne les exprime pas mais constitue une autre réalité discursive qui prend leur place. L'identité de genre exprimée dans le discours n'est donc pas l'expression ou la réalisation plus achevée et optimisée des potentialités ou des capacités d'un individu préalablement identifié, comme lorsqu'il réalise une performance ${ }^{5}$. D'un autre côté, 
il ne s'agit pas d'un masque pris par un acteur le temps d'une représentation par-dessus ou à la place de sa véritable identité. L'identité n'est pas une mascarade, au sens où une fausse identité serait jouée sans que le sujet ne la prenne véritablement ${ }^{6}$. Le discours genré n'est pas l'équivalent d'un vêtement qu'il serait possible de revêtir, sans pour autant que l'apparence ainsi prise n'exprime véritablement l'identité cachée derrière ce masque. Au contraire, l'apparence ainsi prise par l'usage du discours genré définit véritablement et ontologiquement le sujet qui l'assume. Ce n'est pas un rôle théâtral qui s'ajouterait au sexe pré-discursif comme pour lui donner des propriétés supplémentaires artificielles au lieu de les remplacer.

Si l'identité de genre n'est pas ainsi une performance, c'est dans la mesure où le discours genré assumé par le sujet est la condition de possibilité de son identité. Il n'est ni la réalisation ni le masque d'un sujet. Il n'est pas un outil utilisé par un sujet préalablement défini. Si elle parle de pratique discursive ou de discours, ces mots et ces gestes par lesquels le sujet genré existe comme tel apparaissent alors être plutôt de l'ordre de l'histoire au sens défini par le linguiste Émile Benveniste ${ }^{7}$. Il oppose l'histoire qui n'implique pas un engagement du narrateur au discours qui est personnel. Dans la mesure où il n'y a pas de sujet préalable qui assume un genre, il s'agit à proprement parler d'une histoire. Le sujet n'est pas dans un rapport d'extériorité avec l'usage qu'il fait de ce répertoire et de cette grammaire, de sorte qu'il ne peut être défini indépendamment de lui. La grammaire du « discours » portant l'identité de genre d'un sujet se distingue ainsi de celle utilisée dans le langage courant, où un sujet produit un complément au moyen d'un verbe actif, comme si ces sujets précédaient et activaient leurs diverses identifications. Il s'agit là d'une « fiction grammaticale ${ }^{8} »$ supposant un sujet antérieur à son action. Ainsi identifié, l'événement décrit et l'instance du discours qui le décrit est un artifice. Il n'est donc pas possible de définir un présent de l'histoire puisque, pour Émile Benveniste ${ }^{9}$, c'est cette cö̈ncidence qui le définit. L'énonciation par laquelle le sujet assume son genre est de ce point de vue hors de la temporalité du sujet puisqu'elle instaure le point de vue à partir duquel celui-ci se produit. Si la description ou la fiche d'identité d'une personne suppose l'usage de la grammaire courante, il faut avoir conscience qu'elle induit une mécompréhension de la logique à l'œuvre ${ }^{10}$. Ce n'est qu'une fois qu'il assume son histoire genrée de manière rétroactive que le sujet peut mettre en ouvre un discours où il reconnaît ses mots et ses gestes comme étant ceux de l'homme ou de la femme qu'il est. L'identité est toujours suspendue lorsqu' elle s'exprime ${ }^{11}$. 


\section{Réitérer l'énonciation de soi}

Il ne suffit cependant pas de produire performativement une identité genrée, encore faut-il qu'elle continue à définir l'individu qui l'assume. Cette action doit se propager comme son propre écho. Il ne s'agit pas d'un acte singulier, non seulement dans la mesure où tous les sujets assumant un même genre l'effectuent, mais également au sens où un sujet genré doit continuer à le répéter pour le rester. Son existence dans le temps et non seulement comme instant est inséparable de cette construction réitérée assurant sa persistance et sa stabilité. Cette réitération implique que, comme l'observe Émile Benveniste, la séparation entre l'histoire et le discours n'est pas en pratique parfaitement nette : il est possible de passer de l'un à l'autre instantanément sans que cela n'apparaisse et qu'un plan prenne l'autre en charge ${ }^{12}$. Lorsqu'il s'énonce à nouveau, le sujet déjà identifié se remet en jeu. Il repasse ainsi au plan de l'histoire afin de constituer un nouveau récit ${ }^{13}$, mais cette histoire même est assumée par le sujet du récit premier. Judith Butler ne semble pas décrire alors un processus temporel où ces trois moments se succèderaient l'espace d'un instant, mais une dialectique où les plans d'énonciation se chevauchent et deviennent en fait indiscernables. Elle précise que le sujet préalablement constitué est attaché à son identité et qu'il ne la remet en jeu que pour mieux tenter d'y persévérer. Il apparaît comme auteur de la citation, pro-activement lorsqu'il la réitère et rétroactivement lorsqu'il l'a réitérée, bien que son énonciation elle-même le reconstitue tel qu'il est. Il y a non seulement une coïncidence à la fois entre l'événement décrit et l'instance du discours qui le décrit, mais également entre ce présent et l'histoire qui advient. Cette coïncidence entre l'action d'énoncer et l'acte énoncé, remarquée par Émile Benveniste dans le cas des performatifs ${ }^{14}$, permet de définir la possibilité d'un présent historique. La conscience que le sujet a de la continuité de son existence est permise par cette situation.

Si la norme de genre se reproduisait exactement, ce présent aurait la forme de l'éternité. Dans la mesure où la réitération n'est en fait jamais parfaitement réalisée à l'identique, elle permet en même temps d'identifier de manière sui-référentielle les différents moments présents de cette existence où le sujet assume, à chaque fois dans son discours, une histoire différente. Des décalages se produisent inéluctablement à chaque répétition ou citation ${ }^{15}$. L'énonciation du genre se reproduit à chaque instant, de sorte que son itération a lieu à chaque fois dans un nouveau contexte d'énonciation et selon une nouvelle interprétation. Cela modifie à la fois le contenu de ce qui est énoncé et les modalités d'énonciation. Les 
conventions autorisant l'exercice du performatif ne sont donc en fait jamais parfaitement en place, de sorte que les énonciations « rompent» avec leur contexte. La réitération n’est jamais exacte et le genre est donc un idéal impossible à incarner parfaitement. C'est à ce niveau que Judith Butler situe non seulement la possibilité, mais plus encore la nécessité de la subversion. Elle se produit de manière inéluctable et l'enjeu est donc de l'assumer et de l'amplifier plus que de la provoquer volontairement. La dynamique performative est celle d'une réélaboration et d'une subversion de la norme et non celle de sa sortie. Se produisent des changements et des altérations où apparaissent des formes nouvelles qui demandent à être reconnues, et peuvent l'être en renonçant à l'idéal d'une norme se réalisant parfaitement. Si les performatifs échouent, ils n'échouent pas complètement. D’une manière générale, Judith définit la performativité en prenant ses distances avec une double illusion : aussi bien celle d'une énonciation du genre qui réussirait et produirait un genre idéal que celle d'une énonciation qui échouerait nécessairement ${ }^{16}$. Elle les fait jouer ensemble pour penser une énonciation performative qui réussirait à constituer une norme tout en échouant à la réaliser parfaitement.

La conséquence est que l'identité du sujet excède à la fois ce que l'énonciation de la norme de genre autorise et l'image dans laquelle il se reconnaissait au moment de la réitération performative. L'identité de genre est faite des discontinuités dans lesquelles les normes sont réinvesties et matérialisées en décalage avec ce que serait leur réalisation idéale. Les dissonances du genre ne sont donc pas des accidents secondaires. Il y a une prolifération des identités qui sont des déplacements de la norme incorrectement citée. La constance n'est qu'une apparence que Judith Butler qualifie de comédie ${ }^{17}$. Si l'indentification a une substance durable est désirée, elle ne peut jamais être achevée que dans une mise en scène fantasmatique et fictive. C'est ce que permet de comprendre la définition de la fonction de l'énonciation comme performative plutôt que comme constative ou théâtrale Si elle rend compte de la manière dont est produit le sujet, elle permet également de considérer celui-ci comme étant toujours en décalage avec ce qui est attendu de lui. Au contraire, réaliser un objectif ou se mettre dans la peau d'un personnage implique une reproduction du rôle prédéfini.

La définition d'une identité de genre stable aussi bien préalable à son énonciation qu'à sa remise en jeu dans une nouvelle énonciation, ne peut être que rétroactive et en un sens être toujours anachronique, puisqu'elle suppose l'existence d'une essence avant que ses conditions d'existences ne soient réalisées. La manière dont le sujet se reconnaît dans ses actes n'est ainsi 
pas en lui-même un dispositif par lequel le sujet se constitue, mais celui par lequel il prend conscience de la continuité de son existence. Il produit cette conscience rétroactive qui, même si elle est une illusion, le constitue. Judith Butler déplace ainsi la fonction subjectivante de ce que Michel Foucault nomme l'aveu : il n'est pas ce qui instaure le sujet, mais ce qui instaure sa persévérance. Devenu par ses actions autre que lui-même, l'individu reconstitue le récit de son identité pour s'y reconnaitre à nouveau. Il se reconnaît désormais dans ce qui l'a constitué précédemment. Si la stabilité de son identité est minée de l'intérieur par la manière dont elle doit se répéter et par le fait que cette répétition est la mise en récit de décalages, il reconstitue à chaque fois un récit cohérent de lui-même d'une manière présentiste : la cohérence est reconstituée à partir de son identité présente plus qu'elle n'est le constat de ce qu'il a véritablement été. Chacun refait constamment l'expérience de lui-même en se racontant une nouvelle histoire. C'est ainsi que Judith Butler oppose à la performance théâtrale, consistant en l'imitation d'un original, la performativité définie comme une citation sans original faisant comme s'il y avait pourtant un original à reproduire ${ }^{18}$.

\section{La constitution performative de la sexualité : énoncés genrés et énoncés sexuels}

\section{Subsumer la sexualité sous le genre}

L'identité genrée ainsi définie est entre autre « un sens pratique ${ }^{19}$ » du corps et du désir. Si Judith Butler ne thématise pas comme telle la question de la sexualité, la manière dont elle évoque le corps l'inscrit dans la perspective générale de sa théorie de la performativité de l'identité de genre. Le corps y a une place centrale puisque l'énonciation performative du genre passe par des gestes. Les activités sexuelles sont un exemple de tels gestes. Judith Butler n'évoque quasiment pas la sexualité et très peu le corps érogène. Si la question du corps érogène et des pratiques sexuelles n'a pas d'autonomie théorique propre, c'est qu'elle ne renvoie pas à des actes ayant une essence particulière. À une époque où le philosophe Michel Foucault a donné à la sexualité une place centrale pour définir l'identité, cette situation peut étonner ${ }^{20}$. Judith Butler marque en fait un déplacement vers la question du genre dû à deux raisons relativement indépendantes. Il ne s'agit pas de passer à cette nouvelle époque dont Michel Foucault pronostique l'avènement et où la sexualité ne serait plus 
centrale, mais de déplacer et de reformuler cette centralité dans le cadre théorique et politique du féminisme.

D'un point de vue théorique, le genre est une question plus générale qui engage les actions du sujet au-delà de ses pratiques sexuelles et englobe celles-ci. Cette explication apparaît en creux dans la manière dont Judith Butler traite la sexualité comme une énonciation du genre parmi les autres. Elle ne méconnaît pas l'importance que prennent les pratiques sexuelles, mais ce qui est habituellement considéré comme relevant de la sexualité prend place dans un cadre plus large ${ }^{21}$. Cette conception s'inscrit dans l'héritage d'un certain féminisme qu'elle cite d'ailleurs abondamment : lorsque Monique Wittig affirme que les lesbiennes ne sont pas des femmes, elle rabat la sexualité sur le genre. D'un point de vue politique, la mise en avant du genre sur la sexualité provient de la volonté de Judith Butler à s'inscrire dans le champ du féminisme. Il ne s'agit pas, comme pour Monique Wittig, d'affirmer ainsi qu'il est possible d'être autre chose qu' une femme ou un homme, mais de montrer qu'il est possible d'être une femme autrement qu'en ayant une sexualité hétéro-normative. L'enjeu est de libérer le féminisme de l'idée d'une nécessaire base cohérente et unique, plutôt que de multiplier les identités sexuelles justifiant des engagements particuliers ${ }^{22}$. Cela signifie qu'un sujet peut s'énoncer performativement comme femme d'une pluralité de manières, de sorte que cela ajoute un qualificatif ou un prédicat à cette qualité féminine sans pour autant la transformer substantiellement. Les différents actes qui sont alors posés sont de l'ordre des différences qui se produisent lors de la citation de la norme de genre.

\section{L'efficacité performative des pratiques sexuelles}

Les identités sexuelles sont donc envisagées comme des variantes de l'identité de genre. Elles s'inscrivent dans une double logique : à la fois effet de l'identité genrée produite et acte qui la produit. Si Judith Butler ne les lie pas explicitement, elle décrit deux dynamiques qui jouent ensemble. Elles s'articulent de manière logique dans une dialectique comme deux modalités de la même dynamique.

À un premier niveau, les pratiques sexuelles sont l'effet d'un certain corps érogène constitué par identification et nomination avant même l'acte corporel ${ }^{23}$. Avant de s'engager dans des pratiques sexuelles, le sujet est pris dans un ensemble d'énoncés genrés. Il s'identifie à son genre et constitue ses désirs et son corps en fonction de cette identification dans la mesure où une certaine sexualité est normativement associée à un certain genre ${ }^{24}$. Le corps érogène est 
alors l'effet d'une projection plus que d'une action. Ce n'est pas un énoncé sexuel qui produit la sexualité mais un énoncé genré non sexuel qui produit une identité de genre impliquant une certaine sexualité. Le sens du corps érogène est acquis préalablement à toute pratique sexuelle. Judith Butler reformule ainsi l'idée foucaldienne selon laquelle le corps sexué est l'effet d'un énoncé conceptuel qui donne corps et non d'un énoncé gestuel. S'il s'agit bien d'un effet performatif des énoncés genrés, la sexualité n'est pas ainsi performativement constituée au sens où le définit Judith Butler car son énonciation n'est pas sexuelle. Pour reprendre les catégories de John Austin, elle fonctionne selon une logique perlocutionnaire : ce qu' elle produit finalement n'est pas immédiatement ce qui est énoncé mais la conséquence de ce qui est énoncé. Au contraire, la logique performative de l'identité correspond à celle de l'illocutionnaire où l'effet de l'énoncé se confond avec son énonciation. De ce point de vue, lorsqu'il s'engage dans des pratiques sexuelles, le sujet réalise constativement et non performativement son identité de genre.

Cependant, ces actes sont bien en même temps performatifs, dans la mesure où l'énoncé par lequel le sujet persévère dans son être est à la fois un discours personnel et une histoire sans sujet référentiel. Les pratiques sexuelles reproduisent illocutionnairement l'identité de genre à laquelle le sujet est attaché. Ce sont alors des actes sexuels qui produisent une identité sexuelle ${ }^{25}$. L'identité et les pratiques sexuelles ne sont pas la conséquence de l'identité de genre mais la constituent. Le corps s'approprie lui-même la norme de genre. Judith Butler se situe de ce point de vue lorsqu' elle affirme qu'il faut remettre en question l'insistance sur le consentement comme précédant dans tous les cas la sexualité comme s'il était constitutif de la personne ${ }^{26}$. Le consentement est alors l'effet de l'illusion rétroactive de la constance d'une identité toujours déjà présente. Il prend en revanche sens dans la définition de la sexualité comme conséquence d'une identité subjective préalablement définie et identifiée. L'ambiguité vient du fait que les deux logiques fonctionnent en fait toujours ensemble : la conscience que le sujet a de son identité apparaît alors comme l'effet conjugué de son attachement à son identité de genre et à la réitération corporelle d'énoncés sexuels.

\section{Troubler et non contester les genres}

Ces actions ne peuvent que produire des décalages, et non contester l'identité genrée. À la différence de Michel Foucault, elle réinscrit toute pratique sexuelle dans ce cadre. Pour le philosophe, certaines pratiques non-normatives sont plus que des occasions de subvertir les 
identités normativement établies en en ouvrant la signification. Elles interrogent leur existence même et la possibilité de définir une identité. Si le cœur de son travail est d'analyser l'effet de la norme, à plusieurs reprises il ouvre ainsi la possibilité de voir quelque chose d'autre surgir. Judith Butler, au contraire, est attentive à montrer qu'il s'agit d' un fantasme ou d'une illusion ayant une nature similaire et symétrique à celle de la constance parfaite d'une identité produite par un énoncé toujours identique : elle considère qu'il n'est jamais possible de s'affranchir de la norme. Elle fait alors jouer la pensée foucaldienne contre elle-même: contre Michel Foucault évoquant des possibilités de contre-attaques où se produisent une multiplicité de plaisirs excédant toute norme, il s'agit de poser la question foucaldienne de la régulation normative de ces pratiques sans qu'il ne puisse exister d'extériorité à la norme ${ }^{27}$. Judith Butler envisage la possibilité d'un échec de la constitution performative du genre par les pratiques sexuelles, dans la mesure où le corps serait un lieu qui échapperait constitutivement à toute tentative de mise en forme. Elle reprend notamment la manière dont Michel Foucault envisage la sexualité d'Herculine Barbin comme existant hors de toute identité, dans une situation où les catégories de sexe et de genre ont disparu ${ }^{28}$. À d'autres occasions, elle évoque la possibilité selon laquelle le corps serait ce qui est toujours autre, dans la mesure où son érogénéité est imprévisible et peut se recomposer radicalement à chaque expérience sexuelle ${ }^{29}$. Le corps est alors au-delà de ce qu'il énonce. Le site de l'érogénéisation devient multiple et de nouveaux énoncés imprévisibles apparaissent, de sorte que l'identité se trouve prise dans une dynamique de prolifération. Ce transfert n'est pas une reformulation mais une substitution impliquant la possibilité d'autres identités de genre ${ }^{30}$. Quelque chose résiste qui n'est ni un rôle, ni une norme.

Cependant, Judith Butler précise qu'il ne s'agit que d'une illusion d'extériorité irréalisable. Ces pratiques sont invariablement prises en charge par un discours qui produit la sexualité puis dissimule cette production en la désignant comme indomptable et hors de toute norme ${ }^{31}$. Ce qui compte comme extériorité est toujours relatif à un domaine d'énonciation qui autorise et produit cette extériorité. Il réalise cet effet en produisant et en contrôlant un ensemble d'exclusions constitutives. Les pratiques sexuelles excédant la norme ne sont donc pas le surgissement de quelque chose d'impossible. Elles sont comprises par la norme comme étant anormales. Le corps érogène n'est jamais matérialisé hors de la norme, de sorte que les pratiques sexuelles en particulier ne peuvent que la redéployer et non la suspendre ou la transcender. Les pratiques en décalage sont nommées et réintroduites dans la norme. 
Judith Butler s'inscrit dans l'héritage foucaldien en affirmant d'une autre manière que dire oui au sexe, ce n'est pas dire non au pouvoir mais suivre la norme ${ }^{32}$. Elle le reformule pour en faire un effet de la construction de l'identité de genre et l'explique par la nature performative de cette identité. La conséquence est que l'enjeu politique n'est pas de reconnaître une prolifération des identités de genre définies par leurs pratiques sexuelles, mais d'affirmer que les multiples manières d'être homme ou femme, socialement plus ou moins refusées, sont toutes légitimes. Judith Butler s'oppose sur ce point à la perspective cherchant à fonder des identités sexuelles nouvelles en dehors de toute référence au genre, mais établies au nom et à partir d'une jouissance sexuelle. C'est en particulier le cas de la théorie queer qui cherche à reformuler la structure même qui produit les exclusions, alors que Judith Butler est attentive à rendre cette structure dynamique.

\section{Être seul(e) face à la norme}

Une conséquence de cette modélisation des pratiques sexuelles comme énoncés performatifs est la place particulière que Judith Butler donne aux autres individus avec lesquels le sujet est en relation lorsqu'il énonce sexuellement son genre. Ils sont dans une position relative définie par une situation d'observation contraignante. Un énoncé performatif ne peut en effet être efficace que s'il a lieu dans une situation discursive où la forme de l'énonciation ainsi que celui qui l'énonce sont reconnus socialement par un public ${ }^{33}$. L'énonciation du genre est non seulement un acte performatif, mais également un acte relationnel par lequel le sujet se représente face aux autres et devient visible. Il est interpellé par ce public, même s'il n'est pas directement toujours présent : l'impératif d'être virtuellement reconnu est toujours d'actualité. La possibilité de se reconnaître en tant qu'interpellé est constitutive puisqu'elle détermine la réussite de l'énoncé performatif. En un sens, celui-ci est toujours la réponse à une interpellation virtuelle. Les sujets sont ainsi tous pris dans une double relation: ils s'adressent les uns aux autres et reçoivent des adresses les uns des autres. Lorsque Judith Butler considère le rapport aux autres, c'est donc toujours en tant que ces autres sont des agents du pouvoir par lesquels celui-ci exerce sa contrainte. Ils exigent que le sujet soit reconnu selon des normes de genre rigides. Les rapports sociaux possibles sont définis par cette fonction et non pas la possibilité d'un échange : chacun est l'instrument par lequel la norme s'actualise de la manière la plus fidèle possible en tant qu'il adresse aux autres l'exigence de le reconnaître. Il ne discute ni ne négocie le contenu de cette norme, mais 
l'impose. Le sujet est seul face à l'image de lui-même qu'il constitue pour se reconnaître et être reconnu.

Dans ce cadre, les pratiques sexuelles ne sont pas envisagées par Judith Butler comme des relations entre des individus construisant ensemble leurs corps et leurs désirs. Chacun est pour ainsi dire seul face à la norme et se subjective performativement dans une négociation avec elle plutôt que dans un échange avec ses partenaires sexuels. L'énonciation de la norme est cause du corps érogène et du désir. Par opposition, les plaisirs ne sont pas quelque chose qui se produit dans un corps à corps tissé de désirs, mais qui se révèlent dans une identité qui s'énonce. L'autre, avec qui les pratiques sexuelles ont lieu, est ainsi réduit à ce qui le définit dans un rapport structural et fonctionnel, sans que ses caractéristiques propres ne soient en question. Il pourrait s'agir de n'importe quel autre sans intérêt particularisé pour un certain corps ou une certaine psyché, comme si la sexualité était indépendante de son objet. De ce point de vue, Judith Butler s'inscrit dans un héritage structuraliste dont elle ne remet pas en question la logique. Il n'y a donc pas de rapport sexuel, au sens lacanien du terme où, s'il y a du sexe, il ne se noue pas dans un véritable rapport. Il est à ce sujet emblématique que Judith Butler n'évoque jamais ni l'orgasme ni l'amour ${ }^{34}$, qui sont deux formes de cette relation qu'elle n'envisage pas. Elle reste à la limite de ce qui se passe alors dans une performance qui n'est plus la répétition performative de la norme mais un rapport d'échange et d'interpénétration entre des corps et des désirs. Le sujet émerge alors peut-être selon une autre modalité qu'il faudrait définir ${ }^{35}$. Ce serait penser les rapports sexuels comme communication et non plus comme pragmatique : une forme de discours non pas porté sur soi pour s'adresser aux autres, mais offert, partagé et construit avec eux dans une attention plus que dans une intention ${ }^{36}$.

\section{NOTES}

1. Ne seront pris en compte dans ce travail que les trois premiers ouvrages de Judith Butler consacrés directement à la modélisation de genre comme étant produit performativement : Trouble dans le genre. Le féminisme et la subversion de 7'identité, Paris, Éditions La découverte, 2005 ; Ces Corps qui comptent. De la matérialité et des 
Timites discursives du « sexe», Paris, Éditions Amsterdam, 2009 ; Le Pouvoir des mots. Politique du performatif. Paris, Éditions Amsterdam, 2008.

2. Judith Butler, Ces Corps qui comptent, op.cit., p. 14-15.

3. Judith Butler, Trouble dans 7e genre, op.cit., p. 259-271; Judith Butler, Ces Corps qui comptent, op.cit., p. 23-27,117, 227-229; Judith Butler, Le Pouvoir des mots, op.cit., p. $82-83$.

4. Judith Butler, Trouble dans 7e genre, op.cit., p. 71, 270 ; Butler Judith, Ces Corps qui comptent, op.cit., p. 228, 14-19.

5. Véronique Billat, Physiologie et méthodologie de 7'entrainement de la théorie à la pratique, Paris, Éditions De Boeck, 2012, p. 9.

6. Judith Butler, Trouble dans 7e genre, op.cit., p. 131-133; Judith Butler, Ces Corps qui comptent, op.cit., p. 27, 233-236.

7. Émile Benveniste, Problèmes de 7inguistique générale, Paris, Éditions Gallimard, 1966, p. 242 .

8. Judith Butler, Ces Corps qui comptent, op.cit., p. 107.

9. Émile Benveniste, Problèmes de 7inguistique générale, op.cit., p. 262-263 ; Michel Arrivé, "Histoire, discours: retour sur quelques difficultés de lecture », in Linx, $9 \mid 1997$, p. 159-168.

10. Nathanel Wadbled, "Dire quelque chose de soi : 1a théorie du genre de Judith Butler comme autofiction philosophique», in Arnaud Genon et Isabelle Grell (dir.), Lisières de 7'autofiction. Enjeux géographiques, artistiques et politiques, Lyon, Éditions des Presses Universitaires de Lyon, 2016, p. 259-276.

11. Nathanel Wadbled, "Exergue : 1a fiction de soi et 1 'archive de la représentation », in Les Représentations dans 7es fictions 7ittéraires, Tome 2, Par 7es pratiques fictionnel7es, Jean-Marie Kouakou (dir.), Paris, Éditions de l'Harmattan, 2010, p. 201-209.

12. Émile Benveniste, 1966, Problèmes de 7inguistique générale, op.cit., p. 242.

13. J'entends ici le terme récit au sens courant de la mise dans un ordre spécifique de faits, et non comme synonyme de la notion benvenistienne d'histoire ainsi que le définit Gérard Genette, (《Frontières du récit », in Communications n 8, 1966, p. 159)

14. Émile Benveniste, Problèmes de 7inguistique générale, op.cit., p. 273-274.

15. Judith Butler. Trouble dans 7e genre, op.cit., p. 263-265; Judith Butler, Ces Corps 
qui comptent, op.cit., p. 131-134, 244 ; Judith Butler, Le Pouvoir des mots, op.cit., p. $226-237$.

16. Nathanel Wadbled, «Pour un conservatisme progressiste. Conditions et effectivité de 1 'action d'après Judith Butler », op.cit.

17. Judith Butler, Trouble dans le genre, op.cit., p. 239 ; Judith Butler, Ces Corps qui comptent, op.cit., p. 134.

18. Judith Butler, ibid., p. 261.

19. Judith Butler, Le Pouvoir des mots, op.cit., p. 247.

20. Michel Foucault, Histoire de 7 a sexualité. Tome 1, La Volonté de savoir, Paris, Éditions Gal1imard, Col1. «Tel», 1994.

21. Judith Butler, Trouble dans le genre, op.cit., p. 224-226; Anne-Emmanuelle Berger, Le Grand Théâtre du genre. Identité, sexualité et féminisme en « Amérique », Paris, Éditions Belin, 2013, p. 78, 151-154.

22. Judith Butler, Trouble dans 7e genre, op.cit., p. 66, 274-275 ; Judith Butler, Ces Corps qui comptent, op.cit., p. 241-234.

23. Judith Butler, Trouble dans 7e genre, op.cit., p. 168-168, 223-228 ; Judith Butler, Ces Corps qui comptent, op.cit., p. 16, 87-93.

24. Nathanel Wadbled, «Subjectivation et organisation des corps », op.cit., p. 43-59.

25. Judith Butler, Ces Corps qui comptent, op.cit., p. 30 ; Judith Butler, Le Pouvoir des mots, op.cit., p. 222, 236-240.

26. Judith Butler, Le Pouvoir des mots, op.cit., p. 154.

27. Judith Butler, Trouble dans le genre, op.cit., p. 203.

Judith Butler, ibid, p. 202.

Judith Butler, Ces Corps qui comptent, op.cit., p. 72-114,192 ; Judith Butler, Le Pouvoir des mots, op.cit., p. 33-35, 240-241.

30. Nathanel Wadbled, «Subjectivation et organisation des corps », op.cit.

31. Judith Butler, Trouble dans le genre, op.cit., p. 165, 206, 214-215, 242 ; Judith Butler, Ces Corps qui comptent, op.cit., p. 30, 207-210, $119-124,130-131,239-240$.

32. Michel Foucault, Histoire de la sexualité Tome I. Volonté de savoir, op.cit., p. $126,208$.

33. Judith Butler, Trouble dans 7e genre, op.cit., p. 60, 84 ; Judith Butler, Ces Corps qui comptent, op.cit., p. 228 ; Judith Butler, Le Pouvoir des mots, op.cit., p. 55-56, 64 - 68.

34. Elle n’évoque ces question que dans deux travaux qui ne concernent pas directement 
1'identité de genre : lorsqu'elle commente la dialectique hégélienne de la conscience malheureuse (Judith Butler, « Attachement obstiné, assujettissement corporel. Une relecture de la conscience malheureuse de Hegel », in La Vie psychique du pouvoir. L'assujettissement en théories, Paris, Éditions Léo Scheer, 2002) et 1orsqu'el1e s'attache à définir la possibilité d’une éthique (Judith Butler, Le Récit de soi, Paris, Éditions des Presses Universitaires de France, 2007). Ces travaux ouvrent une reconsidération des rapports entre discours, performance et sexualité qu'elle ne suit pas dans ceux concernant directement la question du genre.

35. Il serait par exemple possible de partir de la définition relationnelle que Lynda Hart donne à la fois de la performance et de la sexualité pour définir le type de discours alors en jeu (Lynda Hart, La Performance sadomasochiste. Entre corps et chair, Paris, Éditions EPEL, 2003)

36. Plutôt que celui de John Austin, Le modèle discursif alors convoqué pourrait être celui de la réception, développé par Wolfgang Iser (Wolfgang Iser, L'Acte de 7ecture : théorie de 7 'effet esthétique, Bruxelles, Éditions Mardaga,1985). 